\title{
Survival after Sudden Cardiac Arrest in Hospital
}

\author{
Yoshiyu Takeda, M.D., Junichiro Mifune, M.D., \\ Kuniaki Taga, M.D., Sensyu Hifumi, M.D., \\ Yoshifumi Takahashi, M.D., Seitetsu Yamashita, M.D., \\ Tatsuaki Murakami, M.D., and Takashi Tanaka, M.D.
}

\begin{abstract}
SUMMARY
Although there are many reports on sudden cardiac arrest occurring outside the hospital, little is known about the precise prognostic factors that determine the outcome after cardiopulmonary resuscitation. Clinical information before and immediately after sudden cardiac arrest is frequently incomplete because the event occurs outside the hospital. We studied 90 consecutive patients with sudden and unexpected cardiac arrest who were resuscitated in the general ward of our hospital. Twentyfive $(28 \%)$ were discharged from the hospital. Multivariate analysis revealed that the promptness of initiation of CPR, age, severity of cardiac dysfunction, time and the type of arrhythmia are of significance in relation to survival. To evaluate long-term survival after hospital cardiac arrest, we analyzed long-term follow-up data accumulated during a 16 year period. In the group of 25 patients in our study, there have been a total of 10 deaths $(40 \%)$. Five of the 10 deaths resulted from recurrent cardiac arrest and 1 was a noncardiac death. There was a high rate of recurrence of cardiac arrest in the first year following resuscitation, especially among the cardiomyopathy patients.
\end{abstract}

\section{Additional Indexing Words:}

Sudden cardiac arrest Cardiopulmonary resuscitation Prognostic factor

CINCE the introduction of external cardiac massage in $1960,{ }^{1 \prime}$ cardio$D$ pulmonary resuscitation has frequently been attempted in hospitals and in the community. There have been some reports about bystander-initiated cardiopulmonary resuscitation for sudden cardiac arrest occurring outside the hospital. 2),3)

We studied the characteristics of sudden cardiac arrest (SCA) patients in the hospital and the predictors of outcome. Our objective was to identify factors that may influence the prognosis of sudden cardiac arrest patients.

From the Division of Internal Medicine, Fukui Cardiovascular Center, Fukui, Japan.

Address for reprints: Junichiro Mifune, M.D., Division of Internal Medicine, Fukui Cardiovascular Center, Shinbo 2-228, Fukui 910, Japan.

Received for publication September 5, 1988.

Accepted February 15, 1989. 
We also assessed long-term outcome in patients who had been discharged from hospital.

\section{Patients And Methods}

We evaluated 90 consecutive sudden cardiac arrest patients in the general ward at Fukui Cardiovascular Center who were resuscitated during a 16 year period from 1971 through 1987. Of the 90 subjects, 47 were males and 43 females. Their ages ranged from 15 to 82 years, with a mean age of 61 . For the purpose of this study, sudden cardiac arrest was defined as the sudden and unexpected cessation of circulation resulting in documented loss of consciousness and requiring initiation of cardiopulmonary resuscitation. Also for the purposes of this study, we excluded the critically ill and patients in the intensive care unit. The study included 25 patients who survived and were discharged from hospital.

We used stepwise logistic regression to identify variables that had a significant "independent" relation to outcome, and performed both the chi-square test and mortality table analysis. The severity of congestive heart failure was evaluated according to the criteria of the New York Heart Association (NYHA).

\section{Results}

\section{Clinical course:}

The outcomes of cases in which cardiopulmonary resuscitation (CPR) was performed are shown in Fig. 1. During the 16 year study period, 90 patients had a SCA. After the initial resuscitation, the pulse and blood pressure were restored in 49 of the 90 patients (54\%), and $41(46 \%)$ died. Eighteen of the 49 survivors died within 24 hours after CPR, and another 6 survivors died before leaving the hospital. At 5 years discharge, 14 of the

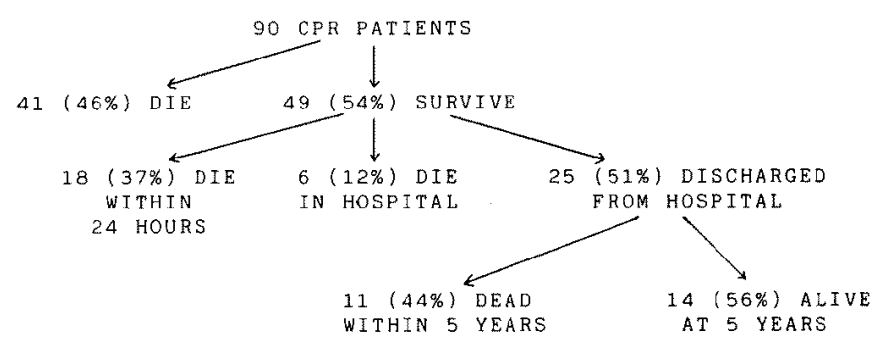

Fig. 1. Outcomes of 90 attempts at resuscitation during the 16 year study period. 
25 remaining patients $(56 \%)$ were still alive.

\section{Clinical characteristics:}

The most common clinical diagnoses in the study group of 90 patients were coronary artery disease $(44 \%)$, cardiomyopathy $(20 \%)$ and valvular disease $(14 \%)$.

The 49 patients in whom CPR was successful were divided into 2 groups. Group I (24 patients, $27 \%$ of the total) was made up of those patients who were resuscitated successfully, survived more than 3 hours, but died before discharge. Group II (25 patients, $28 \%$ of the total) consisted of successfully resuscitated patients who were subsequently discharged from the hospital. Group I will hereafter be referred to as "initial successes", and group II as "long-term successes".

\section{1) Initiation of CiPR}

The interval between the onset of cardiopulmonary collapse and the initiation of CPR was noted accurately. It was the first major prognostic factor for SCA according to stepwise logistic regression multivariate analysis (Table I).

The "early" group, in which the time from collapse to the start of CPR was less than $1 \mathrm{~min}$, showed an initial success rate of $79 \%$ and a longterm success rate of $48 \%$. The "late" group, where the time from collapse to the start of CPR was 10 min or more, had an initial success rate of $20 \%$, but none of the group survived long enough to be discharged from hospital (Table II).

2) Age

Age was the second major prognostic factor in the multivariate analysis (Table I). The older patients (70 years old or more) had a poorer initial

Table 1. Independently Significant Prognostic Factors after Cardiopulmonary Resuscitation by Stepwise Logistic Regression Analysis

\begin{tabular}{l|c|c|c|c}
\hline \multicolumn{1}{c|}{ Factors } & $\begin{array}{c}\text { Estimated } \\
\text { regression } \\
\text { coefficient }\end{array}$ & SE & $\begin{array}{c}\text { Normal } \\
\text { deviation }\end{array}$ & $\begin{array}{c}\text { Significant } \\
\text { probability }\end{array}$ \\
\hline Age & -3.239 & 1.576 & 2.056 & 0.020 \\
Sex & -0.123 & 0.324 & 0.380 & 0.352 \\
Severity of & 0.558 & 0.333 & 1.678 & 0.047 \\
$\quad$ cardiac dysfunction & 0.305 & 0.193 & 1.581 & 0.057 \\
Time & -0.004 & 0.220 & 0.018 & 0.093 \\
Arrhythmia & 1.003 & 3.360 & 1.197 & 0.000 \\
Initiation of CPR & 4.022 & 3.360 & 1.197 & 0.116 \\
QTc & & &
\end{tabular}


success rate $(32 \%)$ than the younger group (age less than $70,57 \%$ ), and the long-term success rate showed the same tendency.

3) Severity of cardiac dysfunction

The severity of cardiac dysfunction before SCA was the third major prognostic factor (Table I). The less severely affected patients, whose NYHA class was I or II, had a better long-term success rate $(45 \%)$ than patients with poorer cardiac function (patients in NYHA class III, 18\%).

4) Time

The time of the cardiac arrest was accurately determined, and was another significant prognostic factor (Table I). The SCA in 20 patients occurred between midnight and 8 a.m., in 43 patients between 8 a.m. and 4 p.m. and in 27 between 4 p.m. and 12 p.m. When all successes and initial failures were compared with regard to the time of the cardiac arrest, a significant difference was seen in the likelihood of survival among the 3 time periods. The likelihood of initial and long-term survival was quite low if the resuscitation took place between midnight and 8 a.m.: only 7 and 3 cases, respectively (Table III).

5) Type of rhythm disturbance

Seventeen patients had ventricular tachycardia, 41 had ventricular fibrillation, and 32 bradycardia or asystole. Arrhythmia was another important prognostic factor (Table I). Those patients who were initial failures were found to have a predominance of asystole. The initial success rate

Table II. Initiation of CPR and Survival

\begin{tabular}{l|c|c|c}
\hline & Initial successes & Long-term successes & Totals \\
\hline CPR $>1$ min & $23^{a}$ & $14^{\mathrm{a}}$ & 29 \\
$\mathrm{GPR} \geq 1<10 \mathrm{~min}$ & $24^{\mathrm{b}}$ & $11^{\mathrm{B}}$ & 46 \\
$\mathrm{CPR} \geq 10 \mathrm{~min}$ & $3^{\mathrm{a}}$ & $0^{\mathrm{r}}$ & 15
\end{tabular}

p Values :

a VS c, d VS f, e VS f: $\mathrm{p}<0.01$.

a VS b, d VS e: $p<0.05$.

b VS c: NS

Table III. Time of Sudden Cardiac Arrest and Survival

\begin{tabular}{l|c|c|c}
\hline & Initial successes & Long-term successes & Totals \\
\hline Midnight to 8 a.m. & $7^{\mathrm{a}}$ & $3^{\mathrm{d}}$ & 20 \\
8 a.m. to 4 p.m. & $25^{\mathrm{b}}$ & $12^{\mathrm{e}}$ & 43 \\
4 p.m. to Midnight & $14^{\mathrm{e}}$ & $9^{\mathrm{f}}$ & 27
\end{tabular}

p Values :

a VS b, a VS c, b VS c, d VS e, d VS f, e VS f: NS. 
was very low in those patients with bradycardia or asystole $(6 \%)$ compared with the $35 \%$ success rate for ventricular tachycardia and the $24 \%$ success rate for ventricular fibrillation (Table IV).

6) Other factors

Other factors, e.g., sex, QTc and basal disease did not influence the prognosis of SCA patients.

Follow-up of long-term survivors:

Adequate follow-up information was available on 25 cases. During the 16 years 12 cases died because of ventricular fibrillation $(\mathrm{N}=3)$, sudden cardiac arrest $(\mathrm{N}=3)$, myocardial infarction $(\mathrm{N}=2)$, heart failure $(\mathrm{N}=3)$, or cerebrovascular accident $(\mathrm{N}=1)$. One of these died 2 months after discharge, 1 after 6 months, and 3 after 12 months. The mean survival time for this group was 3.1 years and the 5 year survival rate was $58 \%$. The prognoses of those patients who had cardiomyopathy were very poor (Fig. 2).

Table IV. Type of Arrhythmia and Survival

\begin{tabular}{c|c|c|c}
\hline & Initial successes & Long-term successes & Totals \\
\hline Ventricular fibrillation & $10^{\mathrm{a}}$ & $14^{\mathrm{d}}$ & 41 \\
Ventricular tachycardia & $6^{\mathrm{b}}$ & $6^{\mathrm{e}}$ & 17 \\
Asystole or bradycardia & $2^{\mathrm{c}}$ & $10^{\mathrm{f}}$ & 32
\end{tabular}

p Values:

a VS b, b VS c: $p<0.01$.

a VS b, d VS e, d VS f, e VS f: NS.

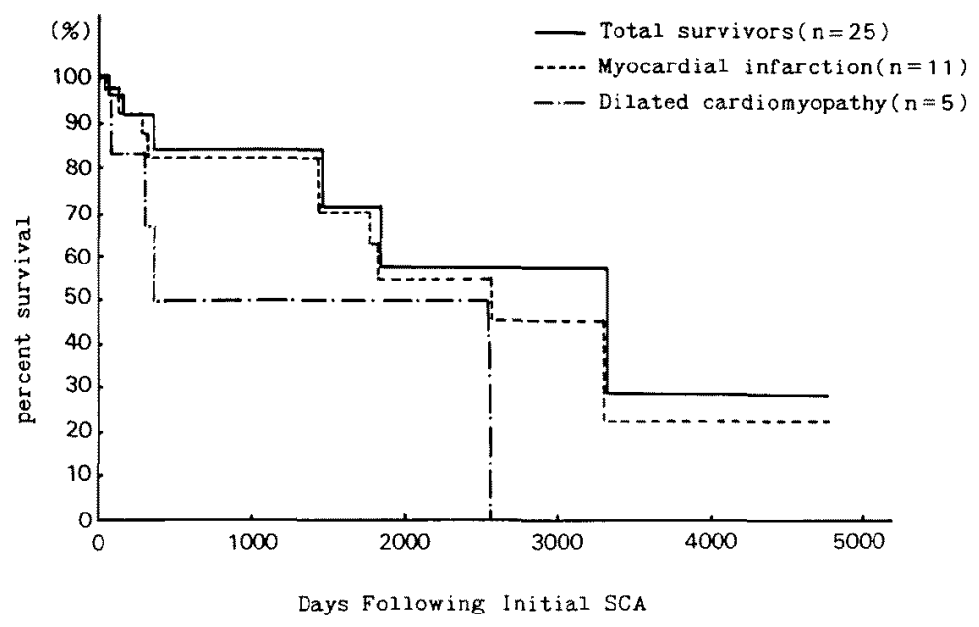

Fig. 2. Survival of sudden cardiac arrest patients. 


\section{Discussion}

Our study was undertaken to identify the characteristics of patients who were resuscitated in the hospital and to compare those who survived and were later discharged with those who died, with respect to these characteristics. Of the patients who underwent cardiopulmonary resuscitation, $26 \%$ were discharged from the hospital, and $56 \%$ of these were still alive 9 years later. Although other papers have reported success rates ranging from under $5 \%$ to over $20 \%,{ }^{4), 5)}$ our high success rate was partially due to the fact that the SCAs occurred inside the hospital.

The interval between the onset of SCA and the initiation of CPR is the most important factor determining the success rate of CPR and the prognosis of survivors of SCA. Bedell et al $^{6)}$ reported that among the 241 patients whose arrest lasted longer than $15 \mathrm{~min}, 95 \%$ died, whereas mortality declined to $44 \%$ overall for patients who were resuscitated within $15 \mathrm{~min}$ after arrest. In our study, the "early" group, whose time from collapse to the start of CPR was less than $1 \mathrm{~min}$, had a long-term success rate of $48 \%$, and the "late" group, in which this time was 10 min or more included no patients who were discharged alive.

Delay in initiating CPR has predictably deleterious effects on the likelihood and quality of survival. Mortality increases with greater delay. An early study showed a high rate of survival in patients resuscitated within 4 min of collapse as opposed to only $5 \%$ survival in those whose CPR was delayed more than 4 min." A recent study corroborated these findings and also suggested that patients resuscitated after $5 \mathrm{~min}$ had more residual CNS impairment. ${ }^{8)}$ Copley $^{91}$ reported that 6 of the 7 who received CPR within $5 \mathrm{~min}$ survived and 5 had no residual CNS impairment, whereas 6 of the 12 experiencing delayed CPR survived, and only 1 had no residual CNS impairment.

The time of the SCA is a major prognostic factor. In our results the initial and long-term success rates were very poor between midnight and 8 a.m. Hollingsworth ${ }^{10}$ ) reports that, of the long-term survivors, only 4 of 29 had cardiopulmonary arrests between the hours of 11 p.m. to 7 a.m. Their failure to survive may have been due to decreased efforts at resuscitation because of delays in reporting, or resuscitation being performed by fewer staff.

Ventricular fibrillation, particularly that accompanying acute myocardial infarction, has been associated with a high survival potential, ${ }^{(1)}$ and asystole has shown a low survival potential. ${ }^{6), 12)}$ Iseri $^{13)}$ reported that of 133 persons with spontaneous cardiac arrest attended by paramedics within 
$10 \mathrm{~min}, 100(75 \%)$ had ventricular fibrillation as the initial rhythm and 33 $(25 \%)$ had extreme bradycardia or asystole; the latter type of arrhythmia was characterized by sinus arrest or severe sinus bradycardia $(90 \%)$ and complete A-V block (10\%). He also stated that despite cardiopulmonary resuscitation and the administration of epinephrine, atropine, isoprotcrenol and sodium bicarbonate, recovery of the sinus and junctional tissues was infrequent. In our study, the majority of those patients who died during or soon after the SCA were found to have asystole. Iseri reported that coronary artery disease was responsible for $70 \%$ of the cases who showed evidence on autopsy of pre-admission brady-asystolic arrest. It is suggested that such an arrest might be due to a) obstruction of a vessel feeding both areas, such as the proximal right coronary artery, which normally supplies the SA node $55 \%$ and the AV node $90 \%$ of the time; b) generalized anoxic depression of both SA and AV sites; c) neurogenic suppression of both nodes, such as might occur with strong vagal stimuli; d) perfusion of both structures with toxic substances such as endogenous metabolites; or e) diffuse degenerative disease of the SA and AV nodes. ${ }^{13)}$ The poor prognosis of brady-asystolic cardiac arrest, however, cannot be explained by these mechanisms alone. In some cases, brady-asystolic cardiac arrest may be a late stage of ventricular fibrillation.

The main basal diseases are atherogenic coronary artery disease (70$71 \%$ ), cardiomyopathy $(8-16 \%)$ and valvular diseases $(7-12 \%){ }^{111,141}$ Hollingsworth ${ }^{10)}$ reported that if all cases with diseases of the cardiopulmonary system are grouped together and compared with the noncardiopulmonary disease group, no significant correlations with survival or mortality rates are seen.

In our study age is one of the major prognostic factors of SCA. Messert and Quglieri,4) and Camarata et al ${ }^{15)}$ also suggested that age is an important determinant of survival after cardiopulmonary resuscitation. However, Bedell et $\mathrm{al}^{\left.\mathbf{l}^{6}\right)}$ suggest that age does not affect the prognosis for survival after resuscitation, nor does it appear to influence adjustment to chronic illness or level of functioning after discharge from hospital.

In our study circulatory failure was the third major prognostic factor of SCA. Bedell et a ${ }^{6}$ ) reported that one important prognostic factor at the initiation of cardiopulmonary resuscitation was circulatory failure, especially as indicated by hypotension. They further stated that among the $110 \mathrm{pa}-$ tients who had hypotension before arrest, only 2 survived ( $2 \%)$, and that of the 87 patients with a urinary output of under $300 \mathrm{ml}$ per 24 hours on the day before the arrest, none survived. Copley ${ }^{9}$ reported that late-resuscitated patients exhibit higher pulmonary artery pressure and lower thermodilution 
cardiac indices, suggesting more left ventricular dysfunction in this group than in early-resuscitated patients, and also that greater s-GOT and CK enzyme elevations in the late-resuscitated group suggest more extensive ischemic muscle injury, probably skeletal as well as myocardial.

\section{Follow-up of long-term survivors:}

In relation to long-term survival after in-hospital sudden arrest, we analyzed follow-up data accumulated over 16 years. In the group of 25 patients discharged from hospital between 1971 and 1986, there have been a total of 10 deaths $(40 \%)$. Five of the 10 deaths were the result of recurrent cardiac arrest; one was a noncardiac death. The mean period from the onset of SCA to death in the nonsurvivors was 3.1 years. Mortality table analysis demonstrated a high rate of recurrence of cardiac arrest in the first year, especially in the group of cardiomyopathy patients. Myerburg et $\mathrm{al}^{16}$ ) reported that 16 of 24 deaths were the result of recurrent cardiac arrest, and that life table analysis demonstrated a $10 \%$ rate of recurrence of cardiac arrest in the first year. Prognostic factors such as circulatory failure and age did not influence the prognosis of long-term survivors.

In conclusion, the important prognostic factors in cases of sudden cardiac arrest in hospital are promptness of initiation of CPR, age, severity of cardiac dysfunction, time of the sudden cardiac arrest and type of rhythm disturbancc. In long-term survival after in-hospital sudden cardiac arrcst, the cardiomyopathy group had a very poor prognosis and died of a recurrence of arrhythmia.

\section{REFERENGES}

1. Kouwenhoven WB, Jude JR, Knickerbocker GG: Closed-chest cardiac massage. JAMA 173: 1064,1960

2. Bachman JW: Cardiac arrest in the community. Postgraduatc Med 76: 85, 1974

3. Skale BT, Miles WM, Heger JJ, Zipes DP, Prystowsky EN: Survivors of cardiac arrest: prevention of recurrence by drug therapy as predicted by electrophysiologic testing or electrocardiographic monitoring. Am J Cardiol 57: 113, 1986

4. Messert B, Quglieri CE: Cardiopulmonary resuscitation perspectives and problems. Lancet 2: 410,1976

5. Johnson AJ, Tanser PH, Ulan RA, Wood TE: Result of cardiac resuscitation in 552 patients. Am J Cardiol 20: 831, 1967

6. Bedell SE, Delbango TL, Cook F, Epstein FH: Survival after cardiopulmonary resuscitation in the hospital. New Engl J Med 309: 569, 1983

7. Pantridge JF: Mobile coronary care. Chest 58: 229, 1970

8. Lund I, Skulberg A : Cardiopulmonary resuscitation by lay people. Iancet 2: 702, 1976

9. Copley DP, Mantle JA, Rogers WJ, Russell RO, Rackley CE: Improved outcome for prehospital cardiopulmonary collapse with resuscitation by bystanders. Circulation 56: 901, 1977 
10. Hollingsworth JH: The results of cardiopulmonary resuscitation. Ann Int Med 71: 459, 1969

11. Thompson RG, Hallstrom AP, Cobb LA: Bystander-initiated cardiopulmonary resuscitation in the management of ventricular fibrillation. Ann Int Med 90: 737, 1979

12. Castagna J, Weil MH, Shubin $\mathrm{H}$ : Factors determining survival in patients with cardiac arrest. Chest 65: 527,1974

13. Iseri LT, Humphrey SB, Siner EJ: Prehospital brady-asystolic cardiac arrest. Ann Int Med 88: 741,1978

14. Roy D, Waxman HL, Kienzle MG, Buxton AE, Manchlinski FE, Josephson ME: Clinical characteristics and long-term follow-up in 119 survivors of cardiac arrest: relation to inducibility at electrophysiologic testing. Am J Cardiol 52: 969, 1983

15. Camarata SJ, Weil MH, Hanashiro PK, Shubin H; Cardiac arrest in the critically ill: a study of predisposing causes in 132 patients. Circulation 44:688, 1971

16. Myerburg RJ, Kessler KM, Estes D, Conde CA, Luceri RM, Zaman L, Kozlovskis PL, Castellanos A: Longterm survival after prehospital cardiac arrest: analysis of outcome during an 8 year study. Circulation 70: 538, 1984 